\title{
Hospitales del Instituto Mexicano del Seguro Social durante los sismos de septiembre de 2017. Análisis desde la perspectiva del Programa Hospital Seguro
}

\author{
Felipe Cruz-Vega, Sandra Elizondo-Argueta, Juan Carlos Sánchez-Echeverría y Jorge Loría-Castellanos
}

Instituto Mexicano del Seguro Social, Dirección de Prestaciones Médicas, División de Proyectos Especiales en Salud, Ciudad de México, México

\begin{abstract}
Resumen
Introducción: La función de los hospitales durante una emergencia mayor o desastre es vital. Su capacidad de respuesta depende de su ubicación geográfica, adecuada organización, seguridad estructural y seguridad de elementos no estructurales. Después de los sismos de septiembre de 2017 se compararon los resultados de las autoevaluaciones y la evaluación del Programa Hospital Seguro en los hospitales inhabilitados pertenecientes al Instituto Mexicano del Seguro Social. Objetivo: Comparar el Índice de Seguridad Hospitalaria (ISH) contra las autoevaluaciones realizadas por las unidades. Método: Se utilizó el ISH y la Lista de Verificación de la Organización Panamericana de la Salud/Organización Mundial de la Salud. La comparación se realizó en tres hospitales inhabilitados posterior a los sismos de septiembre de 2017. Resultados: Se observó variabilidad en los ISH, lo que evidenció sesgos en la autoevaluación: ningún hospital obtuvo una calificación inferior a 0.35 en su ISH aunque tres quedaron inhabilitados en la fase inmediata posterior a los eventos sísmicos. Conclusiones: La variabilidad en los resultados depende de quien aplica el instrumento. La evaluación rápida con ISH proporciona una idea inmediata de la probabilidad de que un hospital continúe funcionando en caso de desastre y permite determinar las acciones de mitigación para incrementar la resiliencia y seguridad de las instalaciones de salud.
\end{abstract}

PALABRAS CLAVE: Resiliencia. Hospital seguro. Sismo.

\begin{abstract}
Introduction: The function of hospitals during major emergency or disaster is vital. Their response capacity depends on their geographic location, adequate organization, structural safety and safety of non-structural elements. After September 2017 earthquakes, self-assessment and Safe Hospital Program assessment results were compared in disabled hospitals belonging to the Mexican Institute of Social Security. Objective: To compare the Hospital Safety Index (HIS) with self-assessments carried out by the units. Method: HIS and the Pan-American Health Organization/World Health Organization checklist were used. The comparison was carried out in 3 hospitals that were disabled after September 2017 earthquakes. Results: Variability was observed in HIS, which revealed self-assessment biases: no hospital obtained a HIS score lower than 0.35, although all 3 were disabled in the immediate phase after the seismic events. Conclusions: Result variability depends on who applies the instrument. Quick HIS assessment provides an immediate idea of the probability for a hospital to continue functioning in case of disaster and allows determining mitigation actions to increase health facilities' resilience and safety.
\end{abstract}

KEY WORDS: Resilience. Safe hospital, Earthquake.

Correspondencia:

Jorge Loría-Castellanos

E-mail: jloriac@ hotmail.com
Fecha de recepción: 17-04-2018

Fecha de aceptación: 18-07-2018

DOI: 10.24875/GMM.18004348
Gac Med Mex. 2018;154:575-581

Disponible en PubMed

www.gacetamedicademexico.com 


\section{Introducción}

La función de los hospitales durante una emergencia o desastre es vital. Su respuesta para la atención de víctimas y evitar pérdida de vidas dependerá no solo de su organización, sino también de que sus instalaciones sean capaces de resistir esa contingencia, es decir, de ser hospitales seguros. ${ }^{1}$

Mantener a salvo al hospital depende de cuatro factores fundamentales: su ubicación geográfica, nivel de la organización, seguridad de la edificación y seguridad de los elementos arquitectónicos no estructurales (líneas vitales, mobiliario y equipo). ${ }^{1}$

De acuerdo con la definición de la Organización Panamericana de la Salud y la Organización Mundial de la Salud (OPS/OMS), un hospital seguro es "un establecimiento de salud cuyos servicios permanecen accesibles y funcionando a su máxima capacidad instalada y en la misma infraestructura, inmediatamente después de un fenómeno destructivo de origen natural, concepto que fue implementado como política nacional de reducción de riesgos por varios países, incluido México, y que permite que los hospitales desde su planeación cuenten con las medidas mínimas indispensables para proteger la vida, la inversión y la función. ${ }^{1}$

Para determinar que un hospital es seguro, un equipo multidisciplinario aplica la lista de verificación ${ }^{1}$ (LV) de la OPS/OMS, herramienta de evaluación rápida, confiable y de bajo costo que proporciona una idea inmediata de la probabilidad de que un establecimiento de salud continúe funcionando en casos de desastre, sin remplazar los estudios de vulnerabilidad de mayor complejidad, obteniéndose el índice de seguridad hospitalaria (ISH).

En esta investigación se analizan los hospitales del Instituto Mexicano del Seguro Social (IMSS) que presentaron daños por los sismos del 7 y 19 de septiembre de 2017 en México y el resultado de su ISH posterior a dos autoevaluaciones y una evaluación del Programa Hospital Seguro (PHS).

\section{Antecedentes del Programa Hospital Seguro de México}

El PHS de México tiene como antecedente la Conferencia Internacional sobre Mitigación de Desastres en Instalaciones de Salud (1996), donde se emitieron recomendaciones y compromisos para promover estrategias de mitigación de desastres en instalaciones de salud. ${ }^{2}$ Posteriormente, la estrategia Hospitales Seguros frente a Desastres fue incluida en el marco de Acción de Hyogo ${ }^{3}$ (2005) y ratificada en el Marco de Sendai ${ }^{4}$ (2015), con lo que se hizo énfasis en la importancia de la reducción de daños e interrupción de servicios y la resiliencia de las instalaciones de salud; la resiliencia se entiende como la capacidad que tiene un sistema, una comunidad o una sociedad expuestos a una amenaza para resistir, absorber, adaptarse, transformarse y recuperarse de sus efectos oportuna y eficientemente, en particular mediante la preservación y la restauración de sus estructuras y funciones básicas por conducto de la gestión de riesgos, de acuerdo con la Oficina de Naciones Unidas para la Reducción del Riesgo de Desastres.

En 2006, México estableció el Comité Nacional de Evaluación, Diagnóstico y Certificación del Programa Hospital Seguro (CNEDCPHS), presidido por la Coordinación Nacional de Protección Civil (CNPC) de la Secretaría de Gobernación, ente rector de la protección civil que tiene las facultades para actuar desde el ámbito legal. El CNEDCPHS está integrado por representantes oficiales de organismos públicos, privados y sociales, lo cual permite una visión global y equilibrada, y es el responsable de las evaluaciones a los hospitales dentro del PHS.

\section{Instituto Mexicano del Seguro Social y el Programa Hospital Seguro}

El Instituto Mexicano del Seguro Social es la institución de seguridad social más grande de Latinoamérica, atiende a más de 75 millones de personas, cuenta en todo el país con más de 6206 unidades médicas, de las cuales 297 hospitales son de segundo y tercer nivel. ${ }^{5}$

Una estrategia prioritaria en esa institución posterior a los sismos de 1985 fue contar con "hospitales seguros"; una de las lecciones aprendidas fue la necesidad de evacuar a 2300 pacientes de los hospitales que integraban el Centro Médico Nacional en la Ciudad de México y que quedaron inhabilitados debido a las afectaciones en las líneas vitales. Después de la evaluación de daños se demolieron los edificios (con altura de 10 a 12 pisos) y se construyeron nuevas edificaciones con cimientos de más de 14 metros de profundidad, no más de seis pisos y tecnología estructural que incorporó disipadores de energía. ${ }^{6}$ En seguimiento a esta estrategia, en 1997 el Instituto firmó un convenio de colaboración técnica con la OPS/OMS para desarrollar un programa de evaluación y diagnóstico de 
Tabla 1. Medidas y plazos de acciones ante los resultados del Índice de Seguridad Hospitalaria en hospitales del Instituto Mexicano del Seguro Social

\begin{tabular}{lcl}
\hline Categoría & $\begin{array}{c}\text { Índice de Seguridad } \\
\text { Hospitalaria }\end{array}$ & Interpretación de resultados (medidas necesarias) \\
\hline A & 0.66 a 1 & $\begin{array}{l}\text { Medidas urgentes } \\
\text { Aunque es probable que el hospital continúe funcionando en caso de desastres, se recomienda } \\
\text { continuar con medidas para mejorar la capacidad de respuesta y ejecutar medidas preventivas en el } \\
\text { mediano y largo plazo, para mejorar el nivel de seguridad ante desastres. }\end{array}$ \\
& $\begin{array}{l}\text { Medidas a corto plazo } \\
\text { B }\end{array}$ & $\begin{array}{l}\text { Se requieren medidas necesarias en el corto plazo, ya que los niveles actuales de seguridad } \\
\text { del establecimiento pueden potencialmente poner en riesgo a los pacientes, el personal y su } \\
\text { funcionamiento durante y después de un desastre. }\end{array}$ \\
& $\begin{array}{l}\text { Medidas a mediano y largo plazo } \\
\text { Se requieren medidas urgentes e inmediatas, ya que los niveles de seguridad del establecimiento } \\
\text { no son suficientes para proteger la vida de los pacientes y el personal durante y después de un } \\
\text { desastre. }\end{array}$ \\
\hline
\end{tabular}

Seguridad estructural

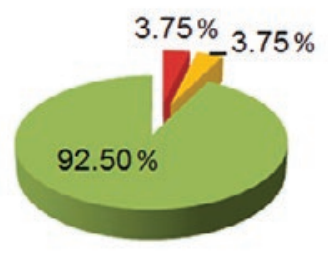

$$
\begin{aligned}
& \text { Bajo } \\
& \text { Medio }
\end{aligned}
$$$$
=\text { Alto }
$$

\section{Seguridad funcional}
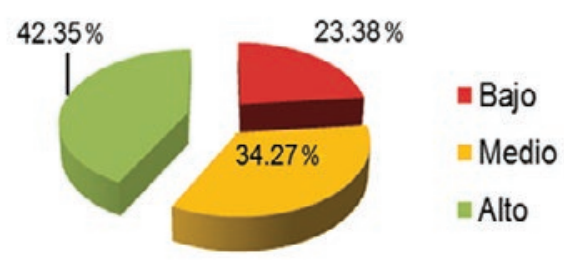

Figura 1. Ejemplo del gráfico con el Índice de Seguridad Hospitalaria.

hospitales e implementar medidas para incrementar su seguridad y fortalecer su infraestructura ante situaciones de desastres. ${ }^{7}$

Al momento de este informe, el CNEDCPHS había evaluado 151 hospitales del Instituto Mexicano del Seguro Social utilizando la LV de la OPS/OMS; una vez realizada la evaluación se aplicó el modelo matemático de la OPS/OMS para determinar el ISH, ${ }^{8}$ el cual clasifica al hospital en las categorías $\mathrm{A}, \mathrm{B}$ y $\mathrm{C}$, que priorizan las necesidades de intervención para el incremento en la seguridad del hospital (Tabla 1 y Figura 1). De los 151 hospitales, cuatro habían sido clasificados en C, tres por vulnerabilidad en la seguridad no estructural y funcional y uno por vulnerabilidad en la seguridad

\section{Seguridad no estructural}

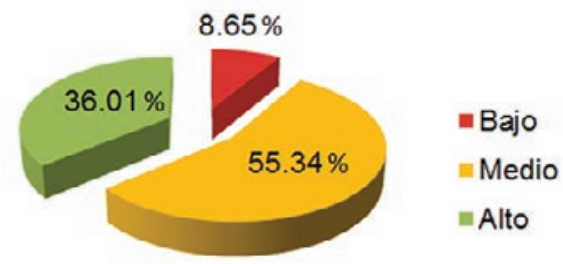

İndice de Seguridad hospitalaria

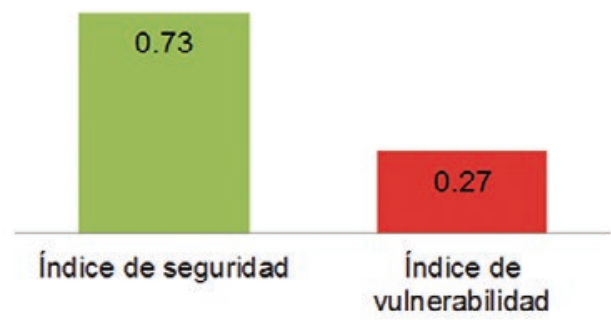

estructural, el cual fue cerrado y demolido; los hospitales con clasificación en A y B mostraron vulnerabilidad en la seguridad no estructural que ha sido mitigada con acciones de reforzamiento, así como vulnerabilidad en la organización funcional, por lo que se implementaron cursos para el personal operativo de atención a la salud y directivo en planes hospitalarios frente a emergencias y desastres y acciones ante diferentes amenazas.

\section{Sismos de septiembre 2017}

Se presentaron dos sismos en septiembre de 2017: el día 7 a las 23:49:18 horas, con magnitud de 8.2 y epicentro a $133 \mathrm{~km}$ al suroeste de Pijijiapan, Chiapas, 
Tabla 2. Hospitales del Instituto Mexicano del Seguro Social con daños por sismos de septiembre de 2017

\begin{tabular}{|c|c|c|c|c|c|c|}
\hline Entidad & $\begin{array}{l}\text { Hospitales por } \\
\text { entidad ( } n)\end{array}$ & $\begin{array}{c}\text { Camas } \\
\text { censables en la } \\
\text { entidad (n) }\end{array}$ & $\begin{array}{l}\text { Hospitales con } \\
\text { daños }\end{array}$ & $\begin{array}{c}\text { Camas } \\
\text { censables en el } \\
\text { hospital (n) }\end{array}$ & $\begin{array}{l}\text { Camas censables } \\
\text { inhabilitadas }(n)\end{array}$ & $\begin{array}{l}\text { Tiempo para } \\
\text { recuperar } 100 \% \text { de su } \\
\text { funcionalidad }\end{array}$ \\
\hline \multirow[t]{2}{*}{ Puebla } & 7 & 728 & $\begin{array}{l}\text { HGR } 36 \text { "San } \\
\text { Alejandro" }\end{array}$ & 415 & 415 & $\begin{array}{l}\text { Construcción nuevo } \\
\text { hospital entre } 2 \text { y } 3 \text { años }\end{array}$ \\
\hline & & & HGZ 5 Metepec & 42 & 0 & 4 meses \\
\hline \multirow{3}{*}{$\begin{array}{l}\text { Ciudad de } \\
\text { México }\end{array}$} & 15 & 2674 & HGR 25 I. Zaragoza & 272 & 272 & 8 meses \\
\hline & & & Gineco-pediatría $3 \mathrm{~A}$ & 103 & 0 & 3 semanas \\
\hline & & & HGZ 32 Villa Coapa & 205 & 205 & 1 año \\
\hline \multirow[t]{2}{*}{ Morelos } & 3 & 394 & HGZ/UMF 7 Cuautla & & & \\
\hline & & & & 52 & 52 & 1 año \\
\hline \multirow[t]{2}{*}{$\begin{array}{l}\text { Estado de } \\
\text { México }\end{array}$} & 17 & 1838 & $\begin{array}{l}\text { HGZ/UMF } 68 \\
\text { Tulpetlac }\end{array}$ & 149 & 0 & 3 semanas \\
\hline & & & $\begin{array}{l}\text { HGR } 196 \text { Fidel } \\
\text { Velázquez }\end{array}$ & 185 & 0 & 3 semanas \\
\hline Total & 43 & 5634 & 8 & 1423 & 944 & \\
\hline
\end{tabular}

HGR = Hospital General Regional, HGZ = Hospital General de Zona, HCZUMF = Hospital General de Zona con Unidad de Medicina Familiar

con afectación a los estados de Oaxaca y Chiapas; ${ }^{9}$ y el día 19 a las 13:14:40 horas, con magnitud de 7.1, epicentro a $12 \mathrm{~km}$ al sureste de Axochiapan, Morelos, y afectación en Morelos, Puebla, Ciudad de México y Estado de México. ${ }^{10}$

\section{Métodos}

Se analizó la información del Instituto Mexicano del Seguro Social sobre los hospitales que resultaron con daños secundarios a los sismos de septiembre de 2017 y los resultados de la evaluación por parte del CNEDCPHS y dos autoevaluaciones realizadas como parte de las acciones rutinarias internas del Instituto.

\section{Resultados}

\section{Impacto de los sismos en la infraestructura hospitalaria del IMSS}

La zona de afectación de ambos sismos incluyó seis estados del país, ninguna unidad médica del Instituto presentó colapso, no hubo muertes de personal, pacientes o visitantes y solo el sismo del 19 produjo daños en algunas unidades hospitalarias.

Derivado de los sismos hubo daños en ocho hospitales ubicados en cuatro entidades del país: Ciudad de México, Estado de México, Puebla y Morelos
(Tabla 2), cuatro con daño inhabilitante (tres cerrados y uno con cierre parcial), lo que representó un déficit de 944 camas (12\%) de las 5634 censables de estas entidades. ${ }^{11}$

Los principales daños en las cuatro unidades que no fueron inhabilitadas estuvieron asociados con elementos arquitectónicos: desprendimiento de plafones, separación en muros de división, grietas en aplanados, ruptura de vidrios, desprendimiento de recubrimientos en las juntas constructivas; la atención médica no fue suspendida y el funcionamiento a $100 \%$ se logró en un periodo de tres semanas a cuatro meses, ya que las unidades cuentan con planes para adecuación de áreas y suspensión de servicios no prioritarios (plan de continuidad de operaciones), un ejemplo fue el Hospital General de Zona 5 de Metepec, Puebla, donde el daño inhabilitó el área de quirófanos y se instalaron dos unidades quirúrgicas móviles.

El Hospital General Regional 25, con 272 camas presentó daños en muros y acabados en el área de hospitalización, que se consideró inhabilitada; la rehabilitación se programó a ocho meses y continúa con función en las áreas de urgencias, consulta externa, imagenología, laboratorio y farmacia.

Los daños en los tres hospitales con inhabilitación total fueron los siguientes:

- Hospital General Regional 36 "San Alejandro", Puebla (415 camas): daño en muros de sostén y 
columnas en los edificios de consulta externa, hospitalización, urgencias, quirófanos y tococirugía; hundimiento y desnivel entre las estructuras.

El hospital tuvo reforzamiento estructural en dos momentos diferentes, primero por daños ocasionados por un sismo en 1993 y posteriormente tras los resultados de una evaluación del PHS. El primer reforzamiento se llevó a cabo con diagonales de acero con disipadores de energía para lograr una mayor seguridad estructural. El segundo reforzamiento también a base de estructura de acero, pero encamisando columnas con placas de acero y dalas diagonales también de acero; la inversión fue de 244.4 millones de pesos y las obra continuaba en desarrollo al momento del sismo de septiembre; el refuerzo contribuyó a que el edificio tuviera una respuesta estructural aceptable y no colapsara.

- Hospital General de Zona 7 Cuautla, Morelos (52 camas): afectación parcial en urgencias, quirófanos, tococirugía y afectaciones mayores en hospitalización.

- Hospital General de Zona 32 Villa Coapa, Ciudad de México (205 camas): daños en muros de carga y acabados, y en escaleras principales y de emergencia.

De los tres hospitales inhabilitados y cerrados, se revisaron los resultados de las evaluaciones (Tabla 3):

1. Evaluación por parte del CNEDCPHS: sigue un protocolo estricto, requiere un mínimo de tres evaluadores capacitados, entrenados y certificados por la Secretaría de Gobernación, deben pertenecer a instituciones diferentes a la que será evaluada, de diferentes disciplinas (médicos, ingenieros, arquitectos, administradores, etcétera); si el protocolo de evaluación no se cumple, la evaluación es cancelada.

2. Autoevaluación Sistema de Información Integral de Protección Civil: en 2012, la CNPC solicitó a las diferentes instituciones de salud que sus unidades médicas ingresaran su información a una plataforma informática donde en forma automatizada se generaba el ISH.

3. Autoevaluación con la Cédula de Identificación de Riesgos Inmobiliarios (CIRI) basada en la LV de la OPS/OMS: en 2016, el Instituto Mexicano del Seguro Social desarrolló una plataforma como parte de las acciones del Programa de Identificación de Riesgos Inmobiliarios, donde las unidades médicas ingresaron su información, con lo que se obtuvo el ISH.
Los tres hospitales en las autoevaluaciones de 2012 obtuvieron un ISH de A, con puntuaciones superiores a las obtenidas en las evaluaciones del CNEDCPHS realizadas por personal capacitado ex profeso para esta actividad; la autoevaluación fue efectuada por personal del hospital. Lo anterior permite considerar la presencia de sesgo al momento de aplicar la LV; al comparar los resultados de las evaluaciones del CNEDCPHS con las autoevaluaciones realizadas en 2016 en los tres hospitales, nuevamente se apreciaron diferencias en las puntuaciones, aunque no tan amplias como en 2012.

Los factores que pueden modificar estos resultados son diversos: la experiencia del personal para aplicar la LV, la comprensión de los conceptos que se evalúan, la forma como se aplica la LV, en la que para la evaluación por el CNEDCPHS se lleva un protocolo estricto y en las autoevaluaciones es el cuerpo de gobierno y los integrantes del Comité de Operaciones de Emergencias.

\section{Discusión}

El Instituto Mexicano del Seguro Social ha adoptado el PHS y se ha comprometido en mitigar los efectos destructivos de los fenómenos adversos e incrementar la seguridad de las instalaciones, ha realizado inversiones para el reforzamiento, ha cerrado hospitales cuando ha sido necesario, los ha reubicado o ha construido nuevas construcciones bajo los lineamientos del PHS; ha demolido un hospital en Morelia, ubicado sobre una falla geológica, así como un hospital en la Ciudad de México con más de 50 años de antigüedad y con daño estructural por el sismo del 20 de marzo de 2012.12

Los tres hospitales inhabilitados y cerrados fueron construidos antes de 1980 cuando existían otros códigos de construcción, modificados después del sismo de 1985; este rubro es evaluado en la LV en el apartado de seguridad estructural que especifica características del terreno, tipo de subsuelo, cómo fue construido y tipo de hospital, entre otros. En una revisión sistemática sobre criterios de riesgo para la selección del sitio de ubicación de un hospital, los principales criterios fueron el costo y la demanda de servicios. ${ }^{13}$ Los hospitales nuevos desde su planeación implementan los lineamientos del PHS, en el que se establece la importancia de la ubicación geográfica, el tipo de suelo, las amenazas a las que se encontrará expuesto y la aplicación de tecnologías adecuadas a las distintas condiciones ambientales del 
Gaceta Médica de México. 2018;154

Tabla 3. Resultados de evaluaciones y autoevaluaciones con base en la Lista de Verificación

\begin{tabular}{|c|c|c|c|c|c|c|}
\hline \multirow[t]{2}{*}{ Entidad } & \multirow[t]{2}{*}{ Hospital } & \multirow{2}{*}{$\begin{array}{c}\text { Inicio de } \\
\text { operaciones }\end{array}$} & \multirow{2}{*}{$\begin{array}{l}\text { Fecha de } \\
\text { evaluación }\end{array}$} & \multicolumn{3}{|c|}{ Categoría/ISH } \\
\hline & & & & $\begin{array}{l}\text { Evaluación } \\
\text { CNEDCPHS }\end{array}$ & $\begin{array}{l}\text { Autoevaluación } \\
\text { SII-PC } 2012\end{array}$ & $\begin{array}{l}\text { Autoevaluación } \\
\text { CIRI } 2016\end{array}$ \\
\hline \multirow[t]{2}{*}{ Ciudad de México } & HGZ 32 Villa Coapa & 1976 & 2007 & $\mathrm{~B}(0.64)$ & $A(0.96)$ & $\mathrm{B}(0.65)$ \\
\hline & & & 2014 & $B(0.59)$ & & \\
\hline Morelos & HGZ/UMF 7 Cuautla & 1968 & 2014 & $A(0.73)$ & $A(0.79)$ & $\mathrm{B}(0.47)$ \\
\hline Puebla & HGR 36 "San Alejandro" & 1972 & 2007 & $\mathrm{~B}(0.62)$ & $A(0.73)$ & $\mathrm{B}(0.56)$ \\
\hline
\end{tabular}

HGR = Hospital General Regional, HGZ = Hospital General de Zona, HGZUMF = Hospital General de Zona con Unidad de Medicina Familiar, CNEDCPHS = Comité Nacional de Evaluación, Diagnóstico, Certificación del Programa Hospital Seguro, SII-PC = Sistema de Información Integral de Protección Civil, CIRI = Cédula de Identificación de Riesgos Inmobiliarios

país que, sin detrimento de la calidad en los servicios médicos, apoyen la sustentabilidad ambiental. Esto implica que al diseñar la unidad médica se consideren factores como reutilización de residuos líquidos y sólidos, minimización de desperdicios y concientización de las ventajas de disminuir el impacto ecológico. ${ }^{11,12}$

Los daños que puede presentar un hospital en el aspecto estructural y no estructural por un sismo son variables, desde problemas de acabados y desprendimiento de plafones o paredes falsas, hasta colapso total de la edificación; el comportamiento estructural durante el sismo influye ampliamente en los componentes no estructurales, sobre todo en los conectados, como las líneas vitales.

Achour et al.14 resumen cómo los daños estructurales y arquitectónicos son diferentes y dependen en forma específica de cada situación; mientras que los daños al equipo y mobiliario suelen ser similares. Estas observaciones se complementan con las del estudio de Kirsh et al..$^{15}$ sobre el impacto en la funcionalidad hospitalaria posterior al sismo de Chile en 2010, en el que ninguno de los siete hospitales estudiados colapsó, sin embargo, los principales daños fueron la falta de suministros externos como agua, electricidad y comunicaciones. En los hospitales del Instituto Mexicano del Seguro Social, ninguno colapsó, sin embargo, hubo daños que disminuyeron su capacidad instalada.

Al analizar los datos de las evaluaciones de los tres hospitales resalta la diferencia en las puntuaciones obtenidas en las autoevaluaciones (realizadas por personal del hospital). Diferentes factores pueden llevar a subestimar o sobreestimar las puntuaciones otorgadas a cada variables respecto a la evaluación del CNEDCPHS, en la cual la subjetividad es eliminada por el protocolo que se sigue y el personal evaluador independiente. En el estudio de Ardalan et al., ${ }^{16} 421$ hospitales de Irán realizaron su autoevaluación en $2015,80 \%$ calificaron con seguridad media y ninguno con alta; los comités de emergencia de los hospitales fueron los responsables del ingreso de la información a la plataforma desarrollada ex profeso. Por su parte, Asefzadeh et al., ${ }^{17}$ al evaluar dos hospitales en Irán, observaron alto nivel de seguridad, sin embargo, sus resultados difieren a los de otros investigadores, lo cual se atribuye a los métodos de recolección de la información: entrevistas orales, cuestionarios escritos, experiencia del recolector de la información, entre otros, que podrían coincidir con los motivos de las diferencias en las tres evaluaciones de los hospitales con daños que referimos.

La vulnerabilidad en la organización funcional es un tema relevante debido a que en ocasiones el hospital se puede mantener física y funcionalmente apto, sin embargo, la falta de preparación del personal para atender una emergencia o desastre puede ser su principal debilidad. En un estudio realizado en Teherán, Irán, ${ }^{18}$ se observó que una de las principales vulnerabilidades en la organización funcional que evalúa la LV estuvo asociada con los planes, guías, procedimientos, monitoreo y evaluación. Chopin et al., ${ }^{19}$ en una investigación efectuada después del sismo de 2007 en Perú, observaron que un mayor número de unidades médicas que contaban con un plan de respuesta a emergencias pudieron mantener la atención médica que aquellas sin un plan ( $p=0.043$ ).

Otro aspecto relevante sobre los planes de respuesta a emergencia es que deben tener un enfoque multiamenaza que considere la atención a diferentes tipos de lesiones, como lo exponen Ushizawa et al. ${ }^{20}$ en relación con la exposición a radiación tras el sismo del 11 de marzo de 2011 en Japón. Al respecto, aunque en este análisis de los hospitales con daños no se evaluó por separado la organización funcional, en el Instituto Mexicano del Seguro Social se aplican protocolos ${ }^{21}$ para atención en emergencias y desastres, que incluyen evacuación de unidades médicas con énfasis en áreas críticas y planes para atención 
a saldo masivo de víctimas, que permiten al personal en forma inmediata adecuar las áreas para la atención de pacientes, suspensión de servicios no prioritarios, establecimiento de áreas de triage y mecanismos de referencia y contrarreferencia.

\section{Conclusiones}

Los sismos de septiembre de 2017 provocaron daños a ocho hospitales sin que ninguno colapsara, sin embargo, tres presentaron daños que los inhabilitaron; en dos se realizan trabajos de reforzamiento y el otro será demolido para construir un nuevo hospital.

La evaluación bajo el estricto protocolo del PHS, independientemente de las autoevaluaciones, permite conocer las medidas que deben efectuarse para fortalecer la seguridad hospitalaria. Los costos que se generen por la inversión en el reforzamiento no tienen comparación con lo que implica salvaguardar la vida.

Después de los sismos de 2017, en México tenemos la oportunidad de seguir desarrollando un sector salud seguro y resiliente.

\section{Bibliografía}

1. Lista de Verificación de Hospitales Seguros. EE. UU.: Organización Panamericana de la Salud; 2008.

2. Conferencia Internacional sobre mitigación de desastres en instalaciones de salud. EE. UU.: Organización Panamericana de la Salud; 1997.

3. Hyogo Framework for Action 2005-2015: Building the Resilience of Nations and Communities to Disasters. EE. UU.: International Strategy for Disaster Reduction; 2005.

4. Sendai framework for disaster risk reduction 2015-2030. EE. UU.: Organización de las Naciones Unidas; 2015.

5. Instituto Mexicano del Seguro Social. Los rostros del IMSS. México: IMSS; 2017.
6. Organización Panamericana de la Salud. Lecciones aprendidas en América Latina en mitigación de desastres en instalaciones de la salud. Aspectos de costo-efectividad. EE. UU.: Organización Panamericana de la Salud/Organización Mundial de la Salud; 1996.

7. Cruz-Vega F, Elizondo-Argueta S, Hernández-Olivas IP, Méndez-Sánchez LM, Saavedra-Gómez JL. Hospital seguro. En: Pérez Castro y Vázquez. Seguridad del paciente al alcance de todos. México: Academia Mexicana de Cirugía/Editorial Alfil; 2013.

8. Índice de seguridad hospitalaria: Guía del evaluador de hospitales seguros. EE. UU.: Organización Panamericana de la Salud; 2008.

9. Servicio Sismológico Nacional. Reporte especial: Sismo de Tehuantepec (2017-09-07 23:49 Mw 8.2). México: UNAM; 2017. Disponible en: http:// www.ssn.unam.mx/sismicidad/reportes-especiales/2017/SSNMX_rep_ esp_20170907 Tehuantepec_M82.pdf

10. Servicio Sismológico Nacional. Reporte especial: Sismo del día 19 de Septiembre de 2017, Puebla-Morelos (M 7.1). México: UNAM; 2017. Disponible en: http://www.ssn.unam.mx/sismicidad/reportes-especiaes/2017/SSNMX_rep esp_20170919 Puebla-Morelos_M71.pdf

11. Instituto Mexicano del Seguro Social. El seguro de México. Los primeros 75 años. México: Algarabía; 2017.

12. Instituto Mexicano del Seguro Social. Informe de labores y programa de actividades 2016-2017. México: Instituto Mexicano del Seguro Social; 2017.

13. Moradian MJ, Ardalan A, Nejati A, Boloorani AD, Akbarisari A, Rastegarfar B. Risk criteria in hospital site selection: a sistematic review. PLoS Curr. 2017;9:pii.

14. Achour N, Miyajima M, Kitaura M, Price A. Earthquake induced structural and non-structural damage in hospitals. Earthquake Spectra. 2011,27(3):617-634

15. Kirsh TD, Mitrani-Reiser J, Bissell R, Sauer LM, Mahoney M, Holmes WT, et al. Impact on hospital earthquake functions following the 2010 Chilean earthquake. Disaster Med Public Health Prep. 2010;4(2):122-128.

16. Ardalan A, Kandi Keleh M, Saberinia A, Khorasani-Zavareh D, Khankeh H, Miadfar J, et al. (2016) 2015 estimation of hospitals safety from disasters in I.R.Iran: the results from the assessment of 421 hospitals. PLoS ONE. 2016;11(9):e0161542.

17. Asefzadeh S, Varyani AS, Gholami S. Disaster risk assessment in educational hospitals of Qazvin based on WHO pattern in 2015. Electronic Physician. 2016;8(1):1770-1775.

18. Ahmadi B, Foroushani AR, Tanha N, Abad AM, Asadi H. Study of functional vulnerability status of Tehran Hospitals in dealing with natural disasters. Electronic Physician. 2016;8(11):3198-3204.

19. Chapin E, Daniels A, Elias R, Aspilcueta D, Doocy S. Impact of the 2007 Ica earthquake on health facilities and health service provision in southern Peru. Prehosp Disaster Med. 2009;24(4):326-332.

20. Ushizawa H, Foxwell AR, Bice S, Matsui T, Ueki $\mathrm{Y}$, Tosaka N, et al. Needs for disaster medicine: lessons from the field of the Great East Japan Earthquake. Western Pac Surveill Response J. 2013:4(1):51-55.

21. Echevarría-Zuno S, Cruz-Vega F, Elizondo-Argueta S, Martínez-Valdés E, Franco-Bey R, Méndez-Sánchez LM. Atención en emergencias y desastres en las unidades de terapia intensiva del Instituto Mexicano del Seguro Social: triage y evacuación. Cir Cir. 2013;81(3):246-255. 